Research Article

\title{
Time-Delayed Acceleration Feedback Control of a Single-Link Flexible Manipulator Using Kalman Filter
}

\author{
Yuming Huang $\mathbb{D}$, Weidong Chen $\mathbb{D}$, and Minqiang Shao $\mathbb{D}$ \\ State Key Laboratory of Mechanics and Control of Mechanical Structures, Nanjing University of Aeronautics and Astronautics, \\ Nanjing 210016, China
}

Correspondence should be addressed to Weidong Chen; chenwd@nuaa.edu.cn

Received 9 November 2020; Revised 23 September 2021; Accepted 26 October 2021; Published 12 November 2021

Academic Editor: Dario Richiedei

Copyright () 2021 Yuming Huang et al. This is an open access article distributed under the Creative Commons Attribution License, which permits unrestricted use, distribution, and reproduction in any medium, provided the original work is properly cited.

\begin{abstract}
The design problem of a discrete controller with time delay and acceleration feedback for a single-link flexible manipulator system is addressed in this paper. The dynamical model of a single-link flexible manipulator system is presented by the adoption of the finite element method and Lagrange's equation. Based on the random-walk process and the discrete reduction method, an augmented discretized delay-free state derivate space equation containing the random noise is established. An acceleration-based Kalman filtering method is developed in order to estimate the system state and external excitation necessary for the controller design. In light of the estimated augmented states, a hybrid controller that combines a feedback control algorithm and a feedforward control algorithm is designed according to optimal control theory and Moore-Penrose theory. Numerical simulation results show that the proposed controller can damp out the vibration response of the flexible manipulator system effectively upon external excitations. Moreover, it is further revealed that the control performance of the presented method can be improved by adding the time delay appropriately.
\end{abstract}

\section{Introduction}

Over the past few decades, manipulators constructed with flexible arms have attracted much more attention because flexible manipulators have the advantages of lighter weight, lower energy consumption, and wider operating range as compared to the traditional rigid manipulators. However, these advantages are generally obtained at the expense of the stiffness of the links. Due to the complexity of link deformation and the rigid-flexible coupling effect [1], establishing an accurate dynamic model and suppressing the unwanted vibration have become a key research challenge in practical design. Comprehensive literature reviews on the dynamic analysis and control schemes of manipulators with flexible links or flexible joints are summarized in a number of survey papers [2-5]. Nevertheless, vibration control is still a key issue in flexible manipulators, especially when the controlled system suffers from a complicated environment.
Various control schemes can be applied to discrete flexible manipulators after the implementation of a series of truncating techniques, such as the finite element method (FEM) [6] or the assumed mode method (AMM) [7]. Based on the FEM and Newmark's solution, Karagulle et al. [8] developed a Matlab code for a two-link manipulator with flexible members and simulated end point vibrations of the system. They designed trapezoidal-velocity-profile inputs to minimize the residual vibrations. Meng et al. [9] presented a stable control strategy to suppress the vibration of a singlelink flexible manipulator built by using AMM. In their work, the performance of the system was improved by optimizing the design parameters of the controller using an online fuzzy genetic optimization algorithm. When the flexible manipulators conduct a space operation, the robust controllers are required to cope up with the unavoidable existence of parameter uncertainties and unknown bounded external interferences. Sayahkarajy et al. [10] proposed a composite controller to control the joint angles and vibrations of a two- 
link manipulator with elastic links, which uses a command preshaping filter as a compensator and uses a multiple-input and multiple-output $\mathrm{H} \infty$ loop-shaping controller as the feedback controller. Yang et al. [11] developed the closedform dynamics of a two-link flexible manipulator under unknown disturbances by using the AMM and Lagrangian methods. A hybrid controller was then designed based on the singular perturbation theory in which an adaptive sliding control was designed for the slow subsystem. Steve et al. [12] proposed an adaptive trajectory control of space flexible joint manipulators under parametric uncertainties and modeling errors. Friction, inertial coupling, and nonlinear characteristics are considered in their dynamic model. Luo et al. [13] designed an active vibration control of flexible manipulators by combining input shaping technique (IS) and an autodisturbance rejection controller (ADRC).

The Kalman filter is a powerful tool to estimate the states of a state-space plant model having the presence of modeling uncertainties or the measurement noise. A linear quadratic Gaussian (LQG) controller associated with the LQR and Kalman-Bucy filter (KBF) is adopted to control the vibration of flexible manipulators by Castri and Messina [14]. They demonstrated that the control spillover and the observation spiller could be reduced by an appropriate design of the regulator and the KBF. Kumar [15] used a positive position feedback (PPF) controller at piezoelectric (PZT) actuators to minimize the end effector vibrations of the flexible link. The negative effect of hardware filters was compensated by a modified Kalman filter. Moreover, in previous works, the accelerometer is generally used to achieve a high vibration feedback control efficiency for the flexible manipulators with good accuracy. The measured acceleration signals can be directly adopted for feedback control to avoid direct current component and trend terms induced by integral actions $[16,17]$. As a result, it is desirable to design an accelerationbased Kalman filter in a straightforward manner for the state observation and control implementation.

In practice, low-frequency vibration is one of the main factors that affect the motion accuracy of space flexible manipulators. From the viewpoint of signal processing, it is necessary to introduce a lower filter in the control structure to eliminate the influence of measurement noise and high frequency signals. As a result, the inevitable time delay induced by the lower filter must be taken into account in the modeling and control of flexible manipulators. Chen et al. [18] was the first to introduce time delay caused by the friction into flexible joint robotic manipulators. $\mathrm{Xu}$ et al. [19-21] conducted a series of studies on the stability and vibration of a flexible joint with transmission delay. $\mathrm{Li}$ and Yin [22] investigated the adaptive position-force control for multiple flexible joint coordinated robot, where the time delays were caused by gear clearance. Although the time delays will bring undesirable control performance or even lead to instability to the system, many researchers have utilized it as a control parameter to improve the efficiency of the controller. A type of time-delayed optimal controller was designed by Qiu and $\mathrm{Wu}$ [23] to compensate for the unknown time delay in a rotating flexible manipulator. A computed torque-time delay approach was presented in
Reference [24] to damp out the end point vibration of a single-link flexible manipulator. According to the measured deflection point, the delay time was chosen to get a fast decay of the end point vibration. Saeed et al. $[25,26]$ proposed a time-delayed position-velocity feedback controller to suppress the vibration of the nonlinear system. They made a clarification of the conditions that the time-delayed controller works as a damper or an exciter. An et al. [27, 28] designed a time-delayed acceleration feedback controller for a smart cantilever beam. The proposed controller not only changed the mass properties of the controlled system but also improved the damping coefficient of the system.

In the present study, a time-delayed acceleration feedback controller considering the effects of the external disturbance is applied to a single-link flexible manipulator. The dynamic model of the control system considering input delay and random excitation was first established by the use of the FEM and Lagrange's equation. The corresponding continuous system is transformed into its discrete delay-free form through a peculiar integral transformation. An augmentation discrete-state derivate space model including system states and external force is constructed, where the unknown external disturbance can be seen to be a randomwalk process. An acceleration-based feedback Kalman filtering equation is then developed to estimate the system state and external force, which are necessary for the controller design. In light of the estimated augmented states, a hybrid controller that combines the feedback control algorithm and the feedforward control algorithm is designed by the use of optimal control theory and Moore-Penrose theory. Numerical simulations are performed to demonstrate the ability of the proposed controller in reducing the vibration response.

\section{Dynamic Modeling}

2.1. Equation of Motion. A schematic diagram of a singlelink flexible manipulator system is shown in Figure 1. The entire system is driven by an electric motor, and a pair of PZT actuators is embedded in the root segment of the flexible link. The mounting base is considered to be a part of the flexible link in the present study. An accelerometer is attached to the distal part of the flexible link, which can be regarded as a payload with mass and inertia.

The link can be maneuvered in the horizontal plane by a revolute joint with one degree of freedom. The rigid motion and the flexible deformations of the link occur in the same plane. The FEM is applied to discretize the flexible link, which satisfies the complicated cross-sectional and boundary conditions. Euler-Bernoulli beam theory is applied to the modeling of flexible links by assuming that the link is long and slender. The coordinate systems are used to develop the dynamic model, as shown in Figure 1. Here, $\mathrm{OXY}$ is the inertial reference frame, and its origin is located at the driving hub. $\mathrm{O}_{1} \mathrm{X}_{1} \mathrm{Y}_{1}$ is the local coordinate system attached to the rigid hub, where $\mathrm{O}_{1} \mathrm{X}_{1}$ is the tangent to the undeformed link. After dividing the flexible link into ne elements, the transverse deflection $w^{i}(x, t)$ at position $x$ in the $i$ th $\left(i \leq n_{e}\right)$ element with respect to its local coordinate 


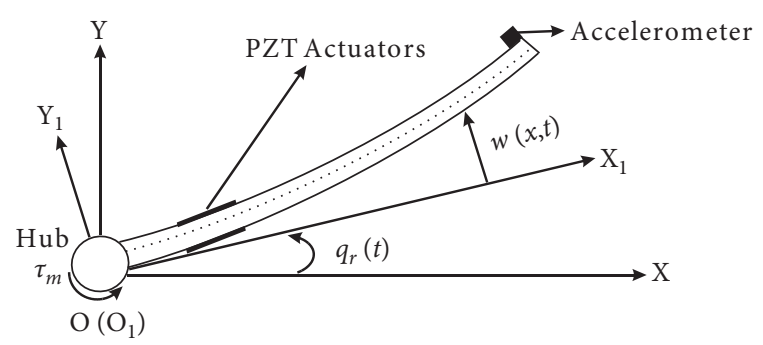

FIgURE 1: Schematic of a single-link flexible manipulator.

system can be expressed in terms of nodal displacement multiplied by shape functions as

$$
w^{i}(x, t)=\mathbf{N}^{i}(x) \mathbf{q}_{f}^{i}(t),
$$

where $\mathbf{N}^{i}(x)$ is the shape function of the $i$ th element, $\mathbf{q}_{f}^{i}=$ $\left[\begin{array}{llll}w^{i} & \theta^{i} & w^{i+1} & \theta^{i+1}\end{array}\right]^{T}$ denotes the deflection and the flexural rotation at nodes $i$ and $i+1$, respectively. The displacement of point $x$ with respect to the inertial frame can be expressed as

$$
\mathbf{r}^{i}(x, t)=\operatorname{Tr}_{0}^{i}(x, t),
$$

where $T$ is the rotational transform matrix from the local frame to the inertial frame, and $\mathbf{r}_{0}^{i}(x, t)=\left[x, w^{i}(x, t)\right]^{T}$ is the displacement of point $x$ at the ith element in the local frame.

Then, the kinetic energy of the flexible link can be written as

$$
T_{f}=\sum_{i=1}^{n_{e}}\left(\frac{1}{2} \int_{0}^{l^{i}} \rho^{i} A^{i}\left(\dot{r}^{i}\right)^{T} \dot{r}^{i} \mathrm{~d} x\right)
$$

where $l^{i}$ is the length of the $i$ th element, and $\rho^{i} A^{i}$ are the density and cross-sectional area of the $i$ th element, respectively.

In addition, the total system kinetic energy includes the kinetic energy of the rotors,

$$
T_{h}=\frac{1}{2} J_{m} \dot{q}_{r}^{2},
$$

and the kinetic energy of the accelerometer,

$$
T_{p}=\frac{1}{2} J_{p}\left(\dot{q}_{r}+\dot{\theta}^{n_{e}+1}\right)^{2}+\frac{1}{2} m_{p} \dot{r}_{p}^{T} \dot{r}_{p},
$$

where $J_{m}$ is the rotary inertial of the hub; $m_{p}$ and $J_{p}$ are the mass and moment of inertia of the payload, respectively; $q_{r}$ is the angular position of the link; and $\theta^{n_{e}+1}$ is the nodal rotation of the $n_{e}$ th element in the flexible link.

The potential energy $V$ is generated only by the elastic deformation of the flexible link in the following form:

$$
V=\sum_{i=1}^{n_{e}}\left(\frac{1}{2} \int_{0}^{l^{i}} E^{i} I^{i}\left(\frac{\partial^{2} w^{i}(x, t)}{\partial x^{2}}\right)^{2}\right),
$$

where $E^{i}$ and $I^{i}$ are Young's modulus and inertial moment of the $i$ th element in the flexible link, respectively.

The work done by the nonconservative external torque $\tau_{m}(t)$ is given by

$$
W=\tau_{m}(t) q_{r}(t)
$$

2.2. Dynamics of PZT Actuators. Two PZT actuators are embedded symmetrically in the mounting base, which is considered as a part of the flexible link. The cross sections are shown in Figure 2. By assuming that $p 1$ and $p 2$ are the nodal numbers of the flexible link on which the actuators act, the bending moments produced by the PZT actuators can be expressed as

$$
M_{p 1}=-M_{p 2}=\bar{c}_{p}\left(w^{p 1}-w^{p 2}\right)+\bar{k}_{v} u,
$$

where $u$ is an externally driven voltage; $\bar{c}_{p}=2 E_{p} A_{p} h_{p}^{2} / l_{p}$; $\bar{k}_{v}=2 E_{p} A_{p} h_{p} k_{v} / l_{p} ; w^{p 1}$ and $w^{p 2}$ are the deflections at nodes $p 1$ and $p 2$, respectively; $E_{p}, A_{p}$, and $l_{p}$ are the Young modulus, cross-sectional area, and length of the piezoelectric actuators, respectively; $h_{p}$ is the distance from the controlling point to the neutral axis of the flexible link; and $\bar{k}_{v}$ is the constant describing the relationship between the control voltage and the deformation of the actuator.

2.3. Dynamics of the Flexible Manipulator. Substituting the expressions for kinetic and potential energy into Lagrange's equations and performing the necessary operations by ignoring the nonlinear terms [29], one obtains the following equations:

$$
\begin{gathered}
m_{r r} \ddot{q}_{r}(t)+\mathbf{m}_{r f} \ddot{\mathbf{q}}_{f}(t)=\tau_{m}(t), \\
\mathbf{m}_{f f} \ddot{\mathbf{q}}_{f}(t)+\mathbf{k}_{f f} \mathbf{q}_{f}(t)+\mathbf{m}_{r f}^{T} \ddot{q}_{r}(t)=\mathbf{b}_{u} u(t-\lambda),
\end{gathered}
$$

where

$$
\left\{\begin{array}{l}
\mathbf{k}_{f f}=\sum_{i=1}^{n_{e}} E^{i} I^{i}\left(\mathbf{B}^{i}\right)^{T}\left(\int_{0}^{l^{i}}\left(\mathbf{N}^{i^{\prime \prime}}(x)\right)^{T} N^{i^{\prime \prime}}(x) \mathrm{d} x\right) \mathbf{B}^{i}, \\
\mathbf{m}_{f f}=m_{p}\left(\mathbf{N}^{i}\left(l^{i}\right) \mathbf{B}^{n_{e}}\right)^{T} \mathbf{N}^{i}\left(l^{i}\right) \mathbf{B}^{n_{e}}+J_{p}\left(\mathbf{N}^{i}\left(l^{i^{\prime}}\right) \mathbf{B}^{n_{e}}\right)^{T} \mathbf{N}^{i^{\prime}}\left(l^{i}\right) \mathbf{B}^{n_{e}}+\sum_{i=1}^{n_{e}} \rho^{i} A^{i}\left(\mathbf{B}^{i}\right)^{T}\left(\int_{0}^{l^{i}}\left(\mathbf{N}^{i}(x)\right)^{T} \mathbf{N}^{i}(x) \mathrm{d} x\right) \mathbf{B}^{i}, \\
\mathbf{m}_{r f}=m_{p} l \mathbf{N}^{i}\left(l^{i}\right) \mathbf{B}^{n_{e}}+J_{p} \mathbf{N}^{i^{\prime}}\left(l^{i}\right) \mathbf{B}^{n_{e}}+\sum_{i=1}^{n_{e}}\left(\rho^{i} A^{i} \int_{0}^{l^{i}}\left(x+(i-1) l^{i}\right) \mathbf{N}^{i}(x) \mathbf{B}^{i} \mathrm{~d} x\right), \\
m_{r r}=m_{p} l^{2}+J_{p}+J_{l},
\end{array}\right.
$$




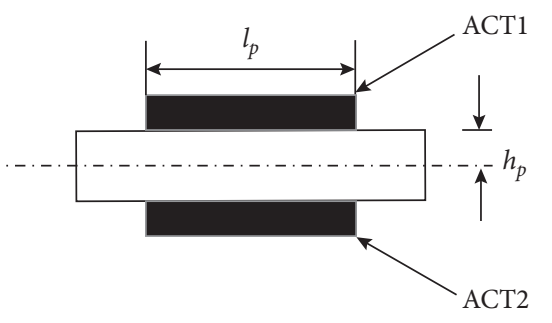

Figure 2: Cross section of the mounting base.

where $J_{l}$ is the equivalent moment of inertia of the flexible link; $\mathbf{B}^{i}\left(i=1 \ldots n_{e}\right)$ is the Boolean operation matrix of the $i$ th element; $\mathbf{b}_{u}$ is the control voltage input matrix; and $\lambda$ is the control delay.

It should be noted that equation (9) describes the rigid motion of the flexible link, while equation (10) represents the vibration of the flexible link subject to control force. Substituting equations (9) into (10) and considering the structural damping, the vibration equation of the flexible link subject to motor torque can be expressed as

$$
\mathbf{m} \ddot{\mathbf{q}}_{f}(t)+\mathbf{c} \dot{\mathbf{q}}_{f}(t)+\mathbf{k} \mathbf{q}_{f}(t)=\mathbf{b}_{\tau} \tau_{m}(t)+\mathbf{b}_{u} u(t-\lambda)
$$

where $\mathbf{m}=\mathbf{m}_{f f}-\mathbf{m}_{r f}^{T} m_{r r}^{-1} \mathbf{m}_{r f}, \mathbf{k}=\mathbf{k}_{f f}$, and $\mathbf{b}_{\tau}=-\mathbf{m}_{r f}^{T} m_{r r}^{-1}$; $c$ is the structural damping matrix, which has the form of the assumed Rayleigh damping; and the corresponding proportional damping coefficient can be determined by a modal test, as done in our previous study [30].

Then, the state-space equation of the flexible manipulator reads

$$
\begin{aligned}
\dot{\mathbf{x}}(t) & =\mathbf{A} \mathbf{x}(t)+\mathbf{B}_{u} u(t-\lambda)+\mathbf{B}_{\tau} \tau_{m}(t), \\
\text { where } & \mathbf{x}=\left[\begin{array}{c}
\mathbf{q}_{f} \\
\dot{q}_{f}
\end{array}\right], \quad \mathbf{A}=\left[\begin{array}{cc}
\mathbf{0}_{n \times n} & \mathbf{I}_{n \times n} \\
-\mathbf{m}^{-1} \mathbf{k} & -\mathbf{m}^{-1} \mathbf{c}
\end{array}\right], \\
\mathbf{B}_{u}=\left[\begin{array}{c}
\mathbf{0}_{n \times 1} \\
\mathbf{m}^{-1} \mathbf{b}_{u}
\end{array}\right], \mathbf{B}_{\tau}=\left[\begin{array}{c}
\mathbf{0}_{n \times 1} \\
\mathbf{m}^{-1} \mathbf{b}_{\tau}
\end{array}\right] . &
\end{aligned}
$$

The acceleration at the tip of link can be expressed as

$$
\mathbf{y}(t)=\mathbf{H} \dot{x}(t),
$$

where $\mathbf{H}=\left[\begin{array}{ll}0 & \mathbf{I}\end{array}\right]$ is the output acceleration matrix and $n=n_{e}$.

2.4. Discrete Delay-Free System. Both the continuous state equation (12) and the measurement (14) are discretized by using a time interval $\Delta \mathrm{T}$. Furthermore, the measurement noise is added to the discretized observation equations by taking into consideration the uncertainties and disturbances in the practical environment. The discrete time forms of system can be written as

$$
\left\{\begin{array}{l}
\mathbf{x}(k+1)=\mathbf{A}_{z} \mathbf{x}(k)+\mathbf{B}_{u z} u(k-h)+\mathbf{B}_{\tau z} \tau_{m}(k), \\
\mathbf{y}(k)=\mathbf{H}_{z} \frac{\mathbf{x}(k+1)-\mathbf{x}(k)}{\Delta T}+\mathbf{v}(k),
\end{array}\right.
$$

where $t=k \Delta \mathrm{T}, x(k), u(k)$, and $\tau_{m}(k)$ are the $k$ th step of the discrete vector $\mathbf{x}(k), \dot{x}(t), u(t)$, and $\tau_{m}(t)$, respectively; the measurement noise vector $\mathbf{v}(k)$ is assumed to be white noise with zero mean, and covariance is $\mathbf{Q}_{v} ; \lambda \cong h \Delta T$ is the relationship between the time delay and the sampling interval, where $h$ is a positive integer; and

$$
\begin{aligned}
\mathbf{A}_{z} & =e^{\mathbf{A} \Delta T}, \\
\mathbf{B}_{u z} & =\int_{(k-1) \Delta T}^{k \Delta T} e^{\mathbf{A}(k \Delta T-\tau)} \mathbf{B}_{u} \mathrm{~d} \tau, \\
\mathbf{B}_{\tau z} & =\int_{(k-1) \Delta T}^{k \Delta T} e^{\mathbf{A}(k \Delta T-\tau)} \mathbf{B}_{\tau} \mathrm{d} \tau, \\
\mathbf{H}_{z} & =\mathbf{H} .
\end{aligned}
$$

A peculiar transformation, as done in common practice [31], is introduced to transform the time-delay system into one which contains no time delay apparently. Let

$$
\left\{\begin{array}{l}
\mathbf{z}(k)=\mathbf{x}(k)+\sum_{m=1}^{h} \mathbf{A}_{z}^{-m} \mathbf{B}_{u z} u(k-h-1+m), \\
\mathbf{z}(0)=\mathbf{x}(0) .
\end{array}\right.
$$

Substituting (16) into (15), the discrete delay-free system can be derived as

$$
\left\{\begin{array}{l}
\mathbf{z}(k+1)=\mathbf{A}_{z} \mathbf{z}(k)+\mathbf{B}_{\mathrm{uzh}} u(k)+\mathbf{B}_{\tau z} \tau_{m}(k), \\
\mathbf{y}(k)=\mathbf{H}_{z} \frac{\mathbf{z}(k+1)-\mathbf{z}(k)}{\Delta T}+\mathbf{v}(k), \\
\mathbf{z}(0)=\mathbf{x}(0)
\end{array}\right.
$$

where $\mathbf{B}_{\mathrm{uzh}}=\mathbf{A}_{z}^{-h} \mathbf{B}_{u z}$.

The random-walk process has been widely used in estimation and identification of unknown time-varying system parameters [32]. The unknown input of the system can be represented to be a random-walk process as follows:

$$
\tau_{m}(k+1)=\tau_{m}(k)+\sigma(k),
$$

where $\sigma(k)$ is a zero-mean white Gaussian random process with covariance $Q_{\sigma}$.

Substituting (19) into (18) yields the following extended discrete delay-free system:

$$
\left\{\begin{array}{l}
\mathbf{z}_{a}(k+1)=\mathbf{A}_{a} \mathbf{z}_{a}(k)+\mathbf{B}_{a} u(k)+\mathbf{D}_{a} \sigma(k), \\
\mathbf{y}(k)=\mathbf{H}_{T}\left(\mathbf{z}_{a}(k+1)-\mathbf{z}_{a}(k)\right)+\boldsymbol{v}(k),
\end{array}\right.
$$


where

$$
\begin{aligned}
\mathbf{z}_{a}(k) & =\left[\begin{array}{c}
\mathbf{z}(k) \\
\tau_{m}(k)
\end{array}\right], \\
\mathbf{A}_{a} & =\left[\begin{array}{cc}
\mathbf{A}_{z} & \mathbf{B}_{\tau z} \\
0 & \mathbf{I}
\end{array}\right], \\
\mathbf{B}_{a} & =\left[\begin{array}{c}
\mathbf{B}_{\mathrm{uzh}} \\
0
\end{array}\right], \\
\mathbf{D}_{a} & =\left[\begin{array}{c}
0 \\
\mathbf{I}
\end{array}\right], \\
\mathbf{H}_{T} & =\frac{1}{\Delta T}\left[\begin{array}{ll}
\mathbf{H}_{z} & 0
\end{array}\right] .
\end{aligned}
$$

\section{Acceleration-Based Kalman Filtering Equation}

In the previous section, the external force is regarded as an auxiliary state into the delay-free state system by using random-walk processes. Therefore, the Kalman filtering method can be used directly to estimate the system state and external force at the same time.

According to Reference [33], the extended discrete delay-free system is a linear deterministic-stochastic one, which can be decomposed into the following linear deterministic system:

$$
\left\{\begin{array}{l}
\mathbf{z}_{1}(k+1)=\mathbf{A}_{a} \mathbf{z}_{1}(k)+\mathbf{B}_{a} u(k), \\
\mathbf{y}_{1}(k)=\mathbf{H}_{T}\left(\mathbf{z}_{1}(k+1)-\mathbf{z}_{1}(k)\right),
\end{array}\right.
$$

and a linear stochastic system,

$$
\left\{\begin{array}{l}
\mathbf{z}_{2}(k+1)=\mathbf{A}_{a} \mathbf{z}_{2}(k)+\mathbf{D}_{a} u(k), \\
\mathbf{y}_{2}(k)=\mathbf{H}_{T}\left(\mathbf{z}_{2}(k+1)-\mathbf{z}_{2}(k)\right)+\boldsymbol{v}(k),
\end{array}\right.
$$

with $\mathbf{z}_{a}(k)=\mathbf{z}_{1}(k)+\mathbf{z}_{2}(k)$ and $\mathbf{y}(k)=\mathbf{y}_{1}(k)+\mathbf{y}_{2}(k)$. It is easy to obtain the solution of $\mathbf{z}_{1}(k)$ in the linear deterministic system by using a transition equation. Hence, it is sufficient to derive the optimal estimate $\widehat{z}_{2}(k)$ of $\mathbf{z}_{2}(k)$ in the stochastic state space (20), so that

$$
\widehat{\mathbf{z}}_{a}(k)=\mathbf{z}_{1}(k)+\widehat{\mathbf{z}}_{2}(k),
$$

becomes the optimal estimate of the state vector $\mathbf{z}_{a}(k)$ in the original system (18).

Assume that a priori estimate of the state vector $\mathbf{z}_{2}(k+1)$ at time $k+1$ satisfies

$$
\widehat{\mathbf{z}}_{2}(k+1 \mid k)=\mathbf{A}_{a} \widehat{\mathbf{z}}_{2}(k) .
$$

Then, the a priori estimate of the measurement can be written as

$$
\widehat{\mathbf{y}}_{2}(k+1 \mid k)=\mathbf{H}_{T}\left(\widehat{\mathbf{z}}_{2}(k+1 \mid k)-\widehat{\mathbf{z}}_{2}(k)\right) .
$$

The residual measurement can be obtained according to (23) and (26) as follows:

$$
\widetilde{\mathbf{y}}_{2}(k+1 \mid k)=\mathbf{y}_{2}(k)-\widehat{\mathbf{y}}_{2}(k+1 \mid k) .
$$

Then, the posteriori state estimate can be corrected using the following expression:

$$
\widehat{\mathbf{z}}_{2}(k+1)=\widehat{\mathbf{z}}_{2}(k+1 \mid k)+\mathbf{K}(k+1) \widetilde{\mathbf{y}}_{2}(k+1 \mid k),
$$

where $\mathbf{K}(k)$ is the Kalman gain matrix.

The error between the actual state and its prediction and the error between the actual state and the updated state estimate are defined at time $k+1$, respectively, as

$$
\left\{\begin{array}{l}
\widetilde{\mathbf{z}}_{2}(k+1 \mid k)=\mathbf{z}_{2}(k+1)-\widehat{\mathbf{z}}_{2}(k+1 \mid k), \\
\widetilde{\mathbf{z}}_{2}(k+1)=\mathbf{z}_{2}(k+1)-\widehat{\mathbf{z}}_{2}(k+1) .
\end{array}\right.
$$

Substituting equation (28) into equation (29) and combining equations (23) and (25), the following state estimation error $\widetilde{z}_{2}(k+1)$ is obtained:

$$
\widetilde{\mathbf{z}}_{2}(k+1)=\left[\mathbf{A}_{a}-\mathbf{K}(k+1) \mathbf{H}_{T} \mathbf{A}_{a}+\mathbf{K}(k+1) \mathbf{H}_{T}\right] \widetilde{\mathbf{z}}_{2}(k)+\left[\mathbf{I}-\mathbf{K}(k+1) \mathbf{H}_{T}\right] \mathbf{D}_{a} \sigma(k)-\mathbf{K}(k+1) \boldsymbol{v}(k),
$$

where equation (30) is a recursive equation for the state estimation error. Choosing the covariance of $\widetilde{z}_{2}(k+1)$ as the optimal objective function and expanding the terms in the equation above, we can express the updated estimate covariance as follows:

$$
\begin{aligned}
\mathbf{P}_{K}(k+1)= & E\left[\widetilde{\mathbf{z}}_{2}(k+1) \widetilde{\mathbf{z}}_{2}^{T}(k+1)\right] \\
= & {\left[\mathbf{A}_{a}-\mathbf{K}(k+1) \mathbf{H}_{T} \mathbf{A}_{a}+\mathbf{K}(k+1) \mathbf{H}_{T}\right] \mathbf{P}_{K}(k)\left[\mathbf{A}_{a}-\mathbf{K}(k+1) \mathbf{H}_{T} \mathbf{A}_{a}+\mathbf{K}(k+1) \mathbf{H}_{T}\right]^{T} } \\
& +\left[\mathbf{I}-\mathbf{K}(k+1) \mathbf{H}_{T}\right] \mathbf{D}_{a} Q_{\sigma} \mathbf{D}_{a}^{T}\left[\mathbf{I}-\mathbf{K}(k+1) \mathbf{H}_{T}\right]^{T}+\mathbf{K}(k+1) \mathbf{Q}_{v} \mathbf{K}^{T}(k+1) .
\end{aligned}
$$


When the derivative of estimate covariance with respect to the Kalman gain matrix equals zero, the trace is minimized. Therefore, the optimal Kalman gain is derived as follows:

$$
\mathbf{K}(k+1)=\frac{\mathbf{A}_{a} \mathbf{P}_{K}(k)\left[\mathbf{H}_{T}\left(\mathbf{A}_{a}-\mathbf{I}\right)\right]^{T}+\mathbf{D}_{a} Q_{\sigma} \mathbf{D}_{a}^{T} \mathbf{H}_{T}{ }^{T}}{\mathbf{H}_{T}\left(\mathbf{A}_{a}-\mathbf{I}\right) \mathbf{P}_{K}(k)\left(\mathbf{A}_{a}-\mathbf{I}\right)^{T} \mathbf{H}_{T}{ }^{T}+\mathbf{H}_{T} \mathbf{D}_{a} Q_{\sigma} \mathbf{D}_{a}^{T} \mathbf{H}_{T}{ }^{T}+\mathbf{Q}_{\nu}} .
$$

In summary, the presented acceleration-based Kalman filtering algorithm can be determined by using the following equations:

$$
\left\{\begin{array}{l}
\mathbf{P}_{0}=E\left[\left(\mathbf{z}_{a}(0)-\widehat{\mathbf{z}}_{a}(0)\right)\left(\mathbf{z}_{a}(0)-\widehat{\mathbf{z}}_{a}(0)\right)^{T}\right] \\
\mathbf{P}_{K}(k+1)=\left[\mathbf{A}_{a}-\mathbf{K}(k+1) \mathbf{H}_{T} \mathbf{A}_{a}+\mathbf{K}(k+1) \mathbf{H}_{T}\right] \mathbf{P}_{K}(k)\left[\mathbf{A}_{a}-\mathbf{K}(k+1) \mathbf{H}_{T} \mathbf{A}_{a}+\mathbf{K}(k+1) \mathbf{H}_{T}\right]^{T} \\
+\left[\mathbf{I}-\mathbf{K}(k+1) \mathbf{H}_{T}\right] \mathbf{D}_{a} Q_{\sigma} \mathbf{D}_{a}^{T}\left[\mathbf{I}-\mathbf{K}(k+1) \mathbf{H}_{T}\right]^{T}+\mathbf{K}(k+1) \mathbf{Q}_{v} \mathbf{K}^{T}(k+1) \\
\mathbf{K}(k+1)=\frac{\mathbf{A}_{a} \mathbf{P}_{K}(k)\left[\mathbf{H}_{T}\left(\mathbf{A}_{a}-\mathbf{I}\right)\right]^{T}+\mathbf{D}_{a} Q_{\sigma} \mathbf{D}_{a}^{T} \mathbf{H}_{T}^{T}}{\mathbf{H}_{T}\left(\mathbf{A}_{a}-\mathbf{I}\right) \mathbf{P}_{K}(k)\left(\mathbf{A}_{a}-\mathbf{I}\right)^{T} \mathbf{H}_{T}^{T}+\mathbf{H}_{T} \mathbf{D}_{a} Q_{\sigma} \mathbf{D}_{a}^{T} \mathbf{H}_{T}^{T}+\mathbf{Q}_{v}}, \\
\widehat{\mathbf{z}}_{a}(0)=E\left(\mathbf{z}_{a}(0)\right), \\
\widehat{\mathbf{z}}_{a}(k+1 \mid k)=\mathbf{A}_{a} \widehat{\mathbf{z}}_{a}(k)+\mathbf{B}_{a} u(k) \\
\widehat{\mathbf{z}}_{a}(k+1)=\widehat{\mathbf{z}}_{a}(k+1 \mid k)+\mathbf{K}(k+1)\left(\mathbf{y}_{a}(k)-\mathbf{H}_{T}\left(\widehat{\mathbf{z}}_{a}(k+1 \mid k)-\widehat{\mathbf{z}}_{a}(k)\right)\right) \\
k=0,1,2, \ldots
\end{array}\right.
$$

\section{Control Design}

In relation to the random-walk process, the system state and input force can be estimated simultaneously by using the Kalman filtering method. Hence, a hybrid controller using the estimated system state and input disturbance force is designed as follows [34]:

$$
u(k)=u_{z}(k)+u_{\tau}(k)=-\mathbf{G}_{z} \widehat{\mathbf{z}}(k)+\mathbf{G}_{f} \widehat{\tau}_{m}(k),
$$

where $\mathbf{G}_{z}$ is the feedback gain with respect to the state estimate and $u_{z}(k)$ is used for suppressing the transient vibration, whereas $\mathbf{G}_{f}$ is the feedforward gain with respect to the external force estimate and $u_{\tau}(k)$ is used for damping the stationary vibration.

Substituting equation (32) into equation (18), we have

$$
\mathbf{z}(k+1)=\left(\left(\mathbf{A}_{z}-\mathbf{B}_{\mathrm{uzh}} \mathbf{G}_{z}\right)\right) \mathbf{z}(k)+\left(\mathbf{B}_{\mathrm{uzh}} \mathbf{G}_{f}+\mathbf{B}_{\tau z}\right) \tau_{m}(k) .
$$

The regular gain $\mathbf{G}_{z}$ can be obtained by using the optimal control method when $\left(\mathbf{A}_{z} \mathbf{B}_{\mathrm{uzh}}\right)$ is controllable. The optimal performance index is defined as follows:

$$
J=\frac{1}{2} \sum_{k=1}^{\infty}\left[\mathbf{z}^{T}(k) \mathbf{Q z}(k)+u_{z}^{T}(k) R u_{z}(k)\right]
$$

where $\mathbf{Q} \geq \mathbf{0}$ and $R \geq 0$ are all symmetric weighting matrices. Then, the feedback gain $\mathbf{G}_{z}$ is given by

$$
\mathbf{G}_{z}=\left(\mathbf{B}_{\mathrm{uzh}}^{T} \mathbf{P B}_{\mathrm{uzh}}+R\right)^{-1} \mathbf{B}_{\mathrm{uzh}}^{T} \mathbf{P} \mathbf{A}_{z}
$$

where $P$ is the solution of the Riccati equation,

$$
\mathbf{A}_{z}^{T} \mathbf{P} \mathbf{A}_{z}-\mathbf{P}-\mathbf{A}_{z}{ }^{T} \mathbf{P} \mathbf{B}_{\mathrm{uzh}}\left(\mathbf{B}_{\mathrm{uzh}}^{T} \mathbf{P B}_{\mathrm{uzh}}+R\right)^{-1} \mathbf{B}_{\mathrm{uzh}}^{T} \mathbf{P} \mathbf{A}_{z}+\mathbf{Q}=0 .
$$

In order to attenuate the effect of the input force, $\mathbf{G}_{f}$ is designed to satisfy the convergence condition $\mathbf{B}_{\mathrm{uzh}} \mathbf{G}_{f}+\mathbf{B}_{\tau z} \longrightarrow \mathbf{0}$, according to the second term on the 
right of (34). There exists the only Moore-Penrose inverse $\mathbf{B}_{\mathrm{uzh}}^{+}$since matrix $\mathbf{B}_{u z}$ has the same size of matrix Buz, which is a row full-rank matrix. According to the theory of Moore-Penrose pseudoinverse, $\mathbf{G}_{f}$ can be obtained from the following equation:

$$
\mathbf{G}_{f}=-\mathbf{B}_{\mathrm{uzh}}^{+} \mathbf{B}_{\tau z}=-\left(\mathbf{B}_{\mathrm{uzh}}^{T}\left(\mathbf{B}_{\mathrm{uzh}} \mathbf{B}_{\mathrm{uzh}}^{T}\right)^{-1}\right) \mathbf{B}_{\tau z} .
$$

In addition, $\mathbf{G}_{f}$ is a least squares solution of minimum norm of $\mathbf{B}_{\mathrm{uzh}} \mathbf{G}_{f}=-\mathbf{B}_{\tau z}$ so that

$$
\left\|\mathbf{B}_{\mathrm{uzh}} \mathbf{G}_{f}+\mathbf{B}_{\tau z}\right\|_{2} \longrightarrow \mathbf{0} .
$$

Finally, the control law with an input delay can be written as

$$
u(k-h)=-\mathbf{G}_{z} \widehat{\mathbf{z}}(k-h)+\mathbf{G}_{f} \widehat{\tau}_{m}(k-h) .
$$

The schematic diagram of the time-delayed acceleration feedback control based on the Kalman filter is shown in Figure 3.

\section{Numerical Simulations}

5.1. Parameters. The applicability of the proposed control strategy has been examined by simulation on a rotating flexible manipulator with variable cross sections, which is simplified from an experimental model with reasonable accuracy [30], as shown in Figure 4. The parameters of the PZT actuator and accelerometer are given in Table 1. According to the parameters in Table 2, the flexible link has been finely meshed to ensure the reliability of the finite element division. In relation to the cross-sectional characteristics, the follow-up unit and the mounting base of the actuators can be divided into five elements, as shown in Figure 5.

In order to achieve higher effective control with a lower computational burden, an iterated improved reduced (IIRS) method is applied [30]. The original model is divided into 20 elements with the dimension of full-order model being 40 . By using the IIRS theory, PZT acting nodes and tip nodes are chosen as master coordinates, while the dimension of the reduced model is finalized to be 4 . The first three eigenvalues and impulse response of the reduced system are compared with that of the original one, as illustrated in Table 3 and Figures 6 and 7, respectively. It can be seen from them that the reduced model has a good agreement with the original model. In addition, the corresponding computational time is decreased from $33 \mathrm{~s}$ to $1 \mathrm{~s}$.

5.2. Effect of Time Delay on Controller. To investigate the effect of the time delay on the proposed controller, the controlled system with zero initial conditions is analyzed upon harmonic excitation containing random white noise. The time delay $\lambda$ is chosen to range from 0 to $6 \Delta \mathrm{T}$, as listed in Table 4, with the state weighting matrix $Q=Q_{0} \times \mathrm{I}_{6} \times 6$, where $Q_{0}=108$, and the control weighting $R=1$. The root mean square (RMS) ratio between the acceleration response of the controlled system ac and the counterpart of the uncontrolled system ao is shown in Figure 8. With the increase in $\lambda$, the RMS ratio is decreased from $10 \%$ to $4 \%$, and it is not nearly altered when $\lambda$ is bigger than $3 \Delta \mathrm{T}$. Hence, a good control performance can be achieved when $\lambda$ is set to be $3 \Delta \mathrm{T}$. It can be found from Figure 9 that the vibration acceleration is further attenuated when the time delay is actively introduced in the proposed controller. These results show that the active utilization of time delay will become a favorable factor in vibration suppression, and the control performance will be improved as the time delay is chosen within a reasonable range [26].

5.3. Effect of Weighting Matrix on Controller. To study the influence of the weighting matrices on the controller, the state weighting Q0 is first selected to range from 100 to 1012 with the control weighting $R$ and time delay $\lambda$ being fixed. The RMS ratio of the acceleration is reduced to $4 \%$ with the increase of Q0 to 108 , and it is not nearly altered when $Q_{0}$ is bigger than 108, as shown in Figure 10 . Therefore, a good control performance can be obtained when $Q_{0}$ is set to be 108 . Subsequently, by changing the control weighting $R$ in the range from 0 to 30 with $Q_{0}=108$ and $\lambda=3 \Delta \mathrm{T}$, the influence of control weighting on the control performance is evaluated, as illustrated in Figure 11. It can be found that the RMS ratio will exhibit larger values with the increase in the control weighting $R$, and the minimum one is achieved when $R=1$, subject to the physical limitation. Finally, a good combination of the state weighting matrix and the control weighting one, which leads to a low RMS ratio, can be found in a 3D colormap surface, as shown in Figure 12.

5.4. Simulation Cases. In order to demonstrate the effectiveness of the proposed method, the controller presented in Section 4 was implemented and verified on a single-link flexible manipulator containing a PZT actuator (as shown in Figure 4) for vibration reduction at its tip node upon different excitations, as listed in Table 5.

A multifrequency torque excitation is first taken into account, which contains the first two resonance frequencies that dominate the vibration response of the structure. By using the proposed acceleration-based Kalman filtering method, the torque excitation along with the system state has been estimated accurately, as indicated in Figures 13 and 14, respectively. The control behavior of the proposed hybrid controller is then assessed by simulation, and the vibration response of the flexible manipulator can be effectively suppressed, as validated in Figure 15. By comparing the result of the hybrid control with that of the control without compensating for an external torque, it can be observed that the former in control performance is clearly superior to the latter, where the RMS ratio of acceleration can be decayed from $10 \%$ to $3.8 \%$, as shown in the subplot of Figure 15.

Subsequently, the torque excitation with a random white noise is considered to verify the robustness of the proposed controller. As mentioned in Section 3, the state and external force are estimated through a Kalman filter 


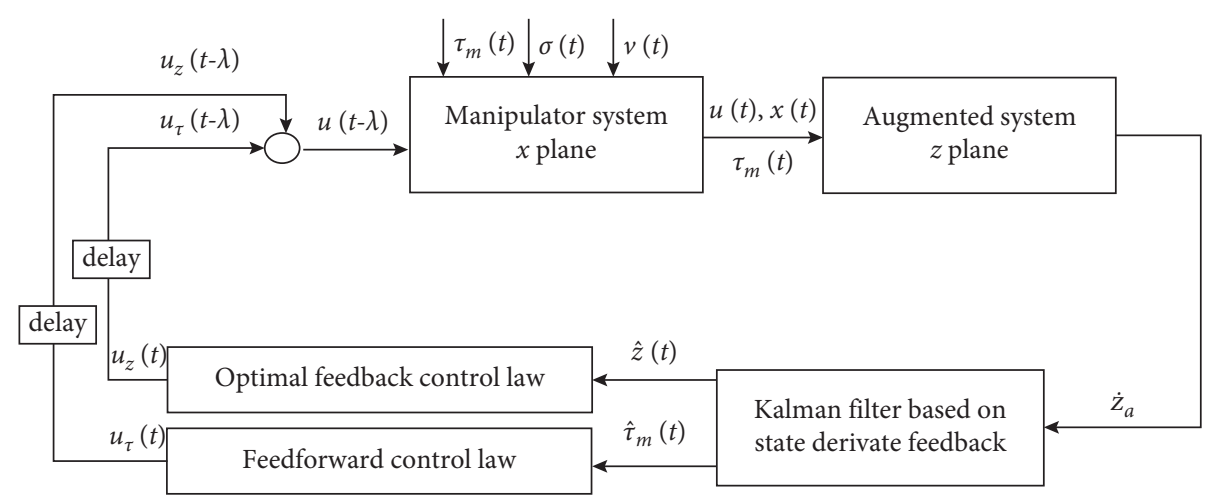

FIGURE 3: Schematic diagram of the control algorithm.

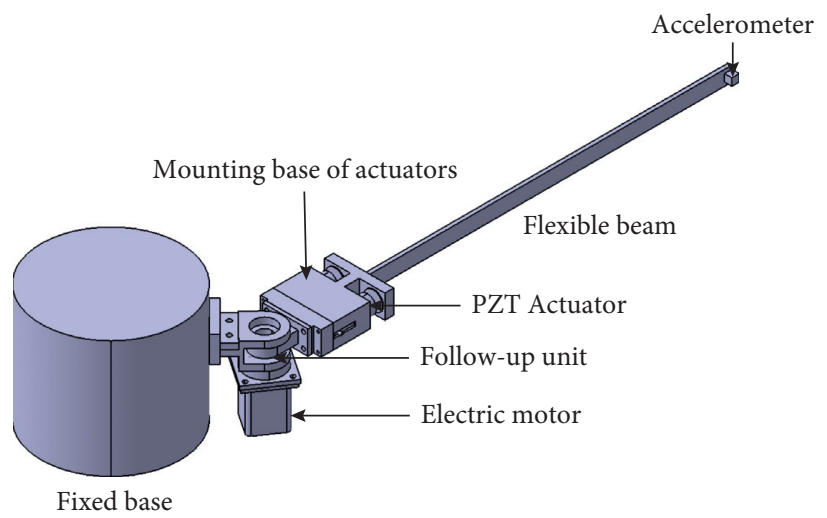

FIGURE 4: Single-link rotating flexible manipulator.

TABle 1: Parameters of the piezoelectric actuator and accelerometer.

\begin{tabular}{|c|c|c|c|c|c|c|}
\hline & $E_{p}(\mathrm{GPa})$ & $A_{p}(\mathrm{~mm})$ & $l_{p}(\mathrm{~mm})$ & $h_{p}(\mathrm{~mm})$ & $m_{p}(\mathrm{~g})$ & $J_{p}$ \\
\hline Piezoelectric actuator & 28.6 & 4902 & 70 & 26 & 4 & $667 \mathrm{gemm} \mathrm{m}^{2}$ \\
\hline
\end{tabular}

TABLE 2: Parameters of elements.

\begin{tabular}{lccccccc}
\hline & $\begin{array}{c}\text { Element } \\
\text { no. }\end{array}$ & $\begin{array}{c}\text { Length } \\
(\mathrm{mm})\end{array}$ & $\begin{array}{c}\text { Cross-sectional } \\
\text { area }\left(\mathrm{mm}^{2}\right)\end{array}$ & $\begin{array}{c}\text { Moment of } \\
\text { inertia }\left(\mathrm{mm}^{4}\right)\end{array}$ & $\begin{array}{c}\text { Mass density } \\
\left(\mathrm{kg} / \mathrm{m}^{3}\right)\end{array}$ & $\begin{array}{c}\text { Young modulus } \\
(\mathrm{GPa})\end{array}$ & $\begin{array}{c}\text { Proportional damping } \\
\text { coefficient }\end{array}$ \\
\hline Follow-up & $\mathrm{e} 1$ & 41 & 525 & $5.3 \times 104$ & & & \\
unit & $\mathrm{e} 2$ & 17 & 2624 & $1.47 \times 106$ & & & \\
Mounting & $\mathrm{e} 3$ & 50 & 1563 & $7.1 \times 105$ & & \\
base & $\mathrm{e} 4$ & 20 & 384 & 4608 & $7.9 \times 103$ & & \\
\multirow{2}{*}{ Flexible link } & $\mathrm{e} 5$ & 12 & 2624 & $1.47 \times 106$ & & $\alpha_{c}=0.1$ \\
\hline
\end{tabular}

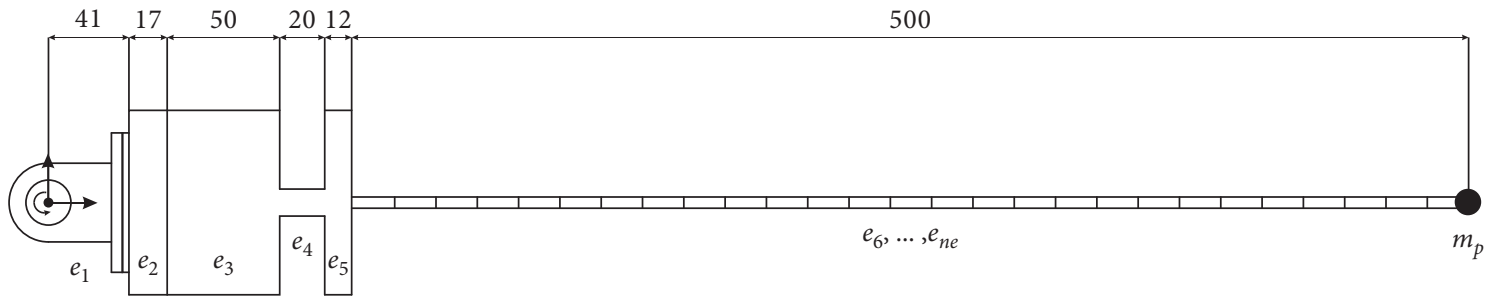

Figure 5: Elements of the flexible link. 
TABLE 3: Comparison of natural frequencies.

\begin{tabular}{lccr}
\hline & & \multicolumn{2}{c}{ Natural frequencies $(\mathrm{Hz})$} \\
& 1 & 14.973 & 3 \\
\hline Original & 3.099 & 14.975 & 33.683 \\
Reduced & 3.099 & 0.0136 & 33.764 \\
Error (\%) & 0.0022 & 0.2388 \\
\hline
\end{tabular}

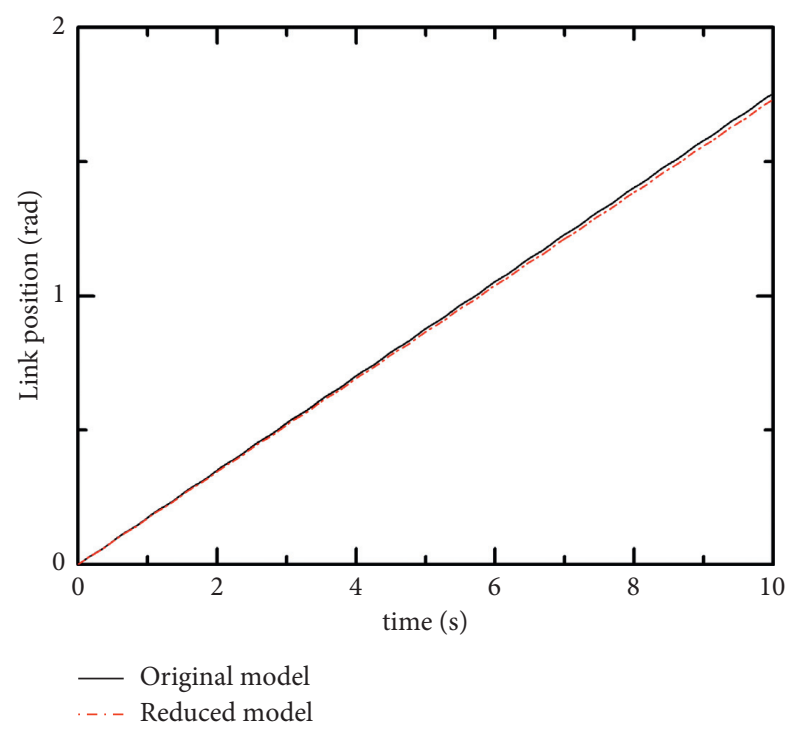

FIgURE 6: Time histories of the link position.

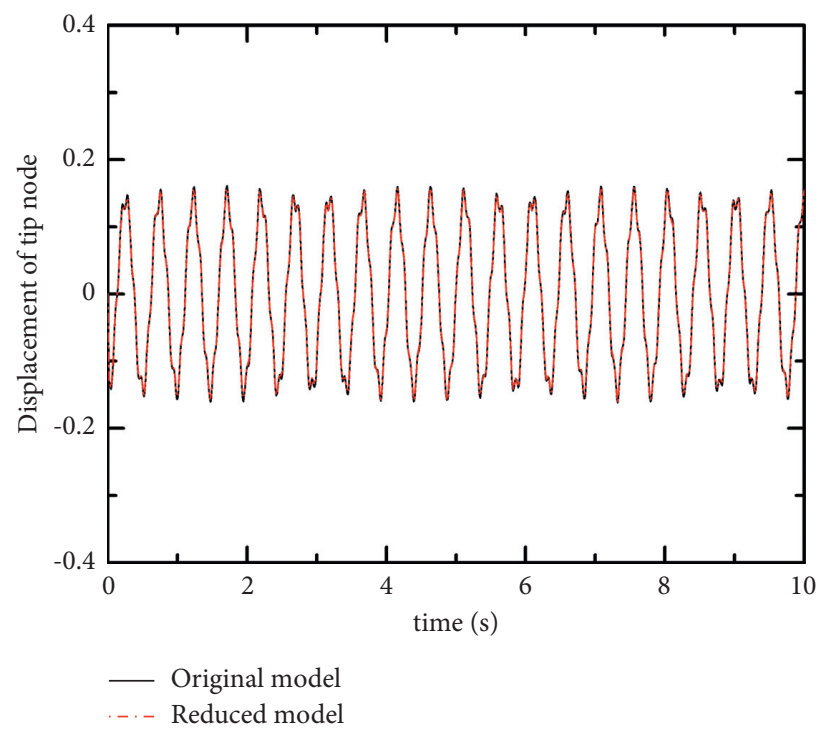

Figure 7: Tip transverse displacement response.

TABLE 4: RMS ratio between $a_{c}$ and $a_{o}$ at different time delays.

\begin{tabular}{lc}
\hline Time delay $(\times \Delta \mathrm{T})$ & Ratio $(\%)$ \\
\hline 0 & 10.39 \\
1 & 5.06 \\
2 & 4.26 \\
3 & 4 \\
4 & 3.99 \\
5 & 4.01 \\
6 & 4 \\
\hline
\end{tabular}

being designed in the state derivate space, and as a result, the robustness of the control system is guaranteed. It is easy to observe that the estimated state and external torque with the noise are in reasonable agreement with the actual counterparts, as shown in Figures 16 and 17, respectively. In turn, the controller can keep the vibration response at tip node converging to a low level, as validated in Figure 18, and the stability of the control system is enhanced under uncertain random disturbance. 


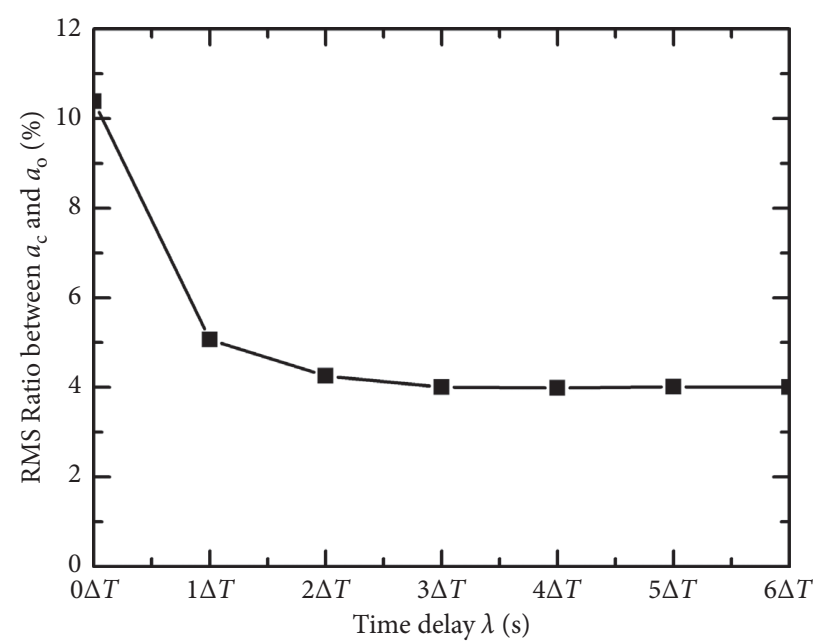

Figure 8: RMS ratio between ac and ao at different time delays $\left(Q_{0}=108, R=1\right)$.

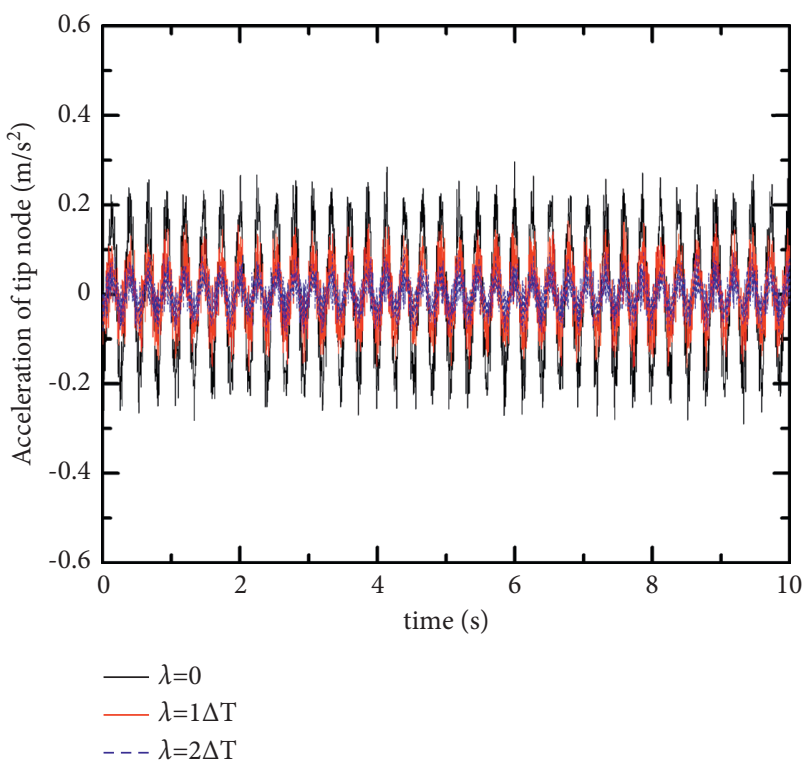

Figure 9: Time histories of the controlled acceleration at the tip node at different time delays.

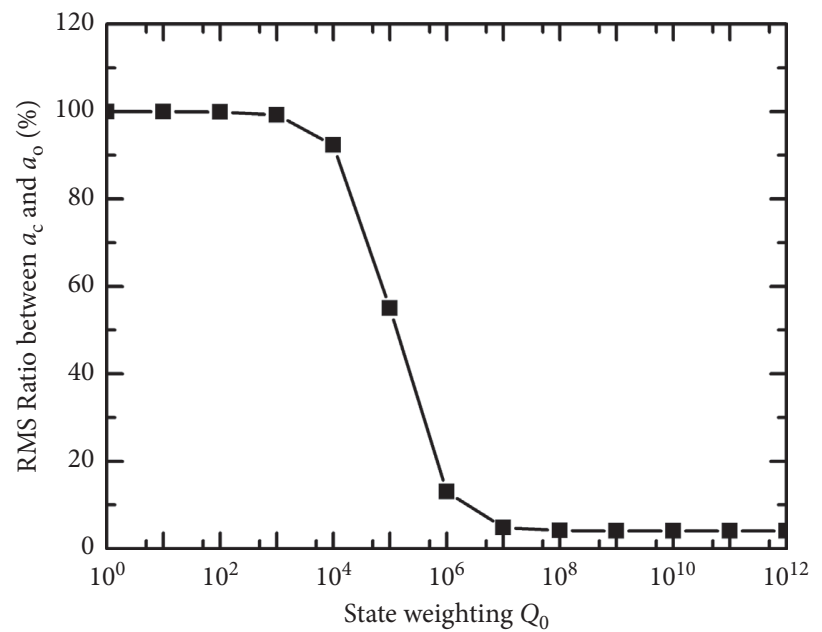

Figure 10: RMS ratio between $\mathrm{a}_{\mathrm{c}}$ and $\mathrm{a}_{\mathrm{o}}$ with respect to $Q_{0}(R=1, \lambda=3 \Delta \mathrm{T})$. 


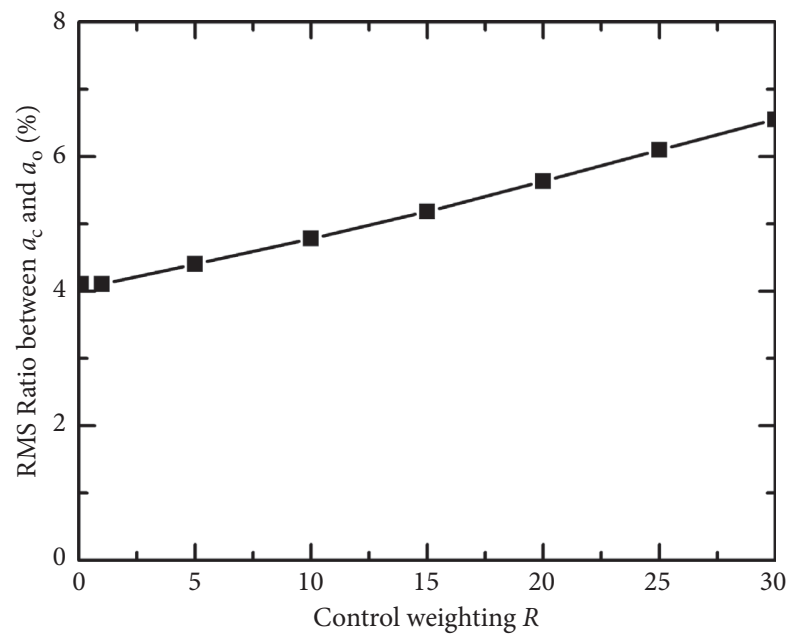

FIgURE 11: RMS ratio between $\mathrm{a}_{\mathrm{c}}$ and $\mathrm{a}_{\mathrm{o}}$ with respect to $R\left(Q_{0}=108, \lambda=3 \Delta \mathrm{T}\right)$.

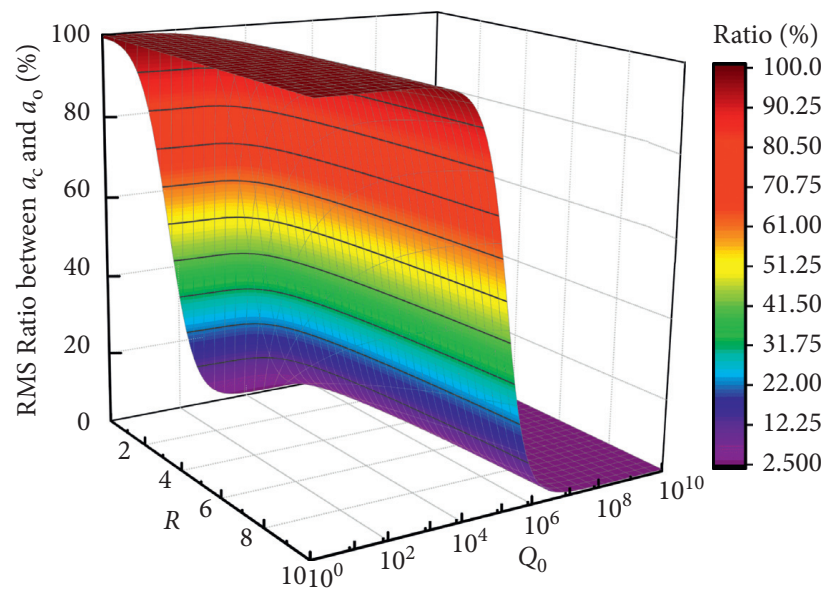

FIgURE 12: RMS ratio between ac and ao with respect to $Q_{0}$ and $R$.

TABLe 5: External excitations.

\begin{tabular}{lcc}
\hline Forms of torque & Mathematical expression & No. of figures \\
\hline Multifrequency excitation & $\tau_{m}(t)=0.1 \sin \left(2 \pi f_{1} t\right)+0.1 \sin \left(2 \pi f_{2} t\right)$ & Figures 13-15 \\
Excitation with noise & $\tau_{m}(t)=0.1 \sin \left(2 \pi f_{1} t\right)+0.1 \sin \left(2 \pi f_{2} t\right)+\sigma(t)$ & Figures 16-18 \\
Input trajectory & $q_{r}(t)=q_{r}\left(t_{0}\right)+\left(6 t^{5} / t_{d}^{5}-15 t^{4} / t_{d}^{4}+t^{3} / t_{d}^{3} 10\right)\left(q_{r}\left(t_{d}\right)-q_{r}\left(t_{0}\right)\right)$ & Figures 19 and 20 \\
\hline
\end{tabular}

$f_{1}$ and $f_{2}$ are the first two resonance frequencies of the flexible manipulator and $\sigma(t)$ is the random noise.

In final, a quintic polynomial trajectory, which is proved to be smoother for tracking purpose [35], is chosen as the external motion excitation of the flexible link in the simulation, as depicted in Figure 19. The vibration response of the flexible manipulator subject to this excitation can be further reduced by the use of the proposed controller, as shown in Figure 20. 


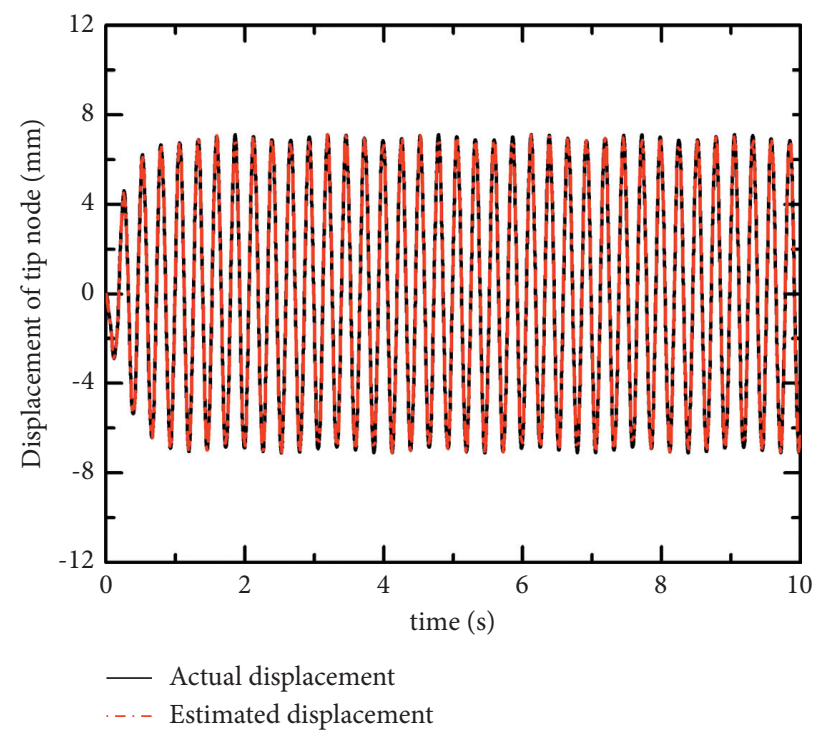

Figure 13: Time histories of displacements at the tip node (multifrequency excitation).

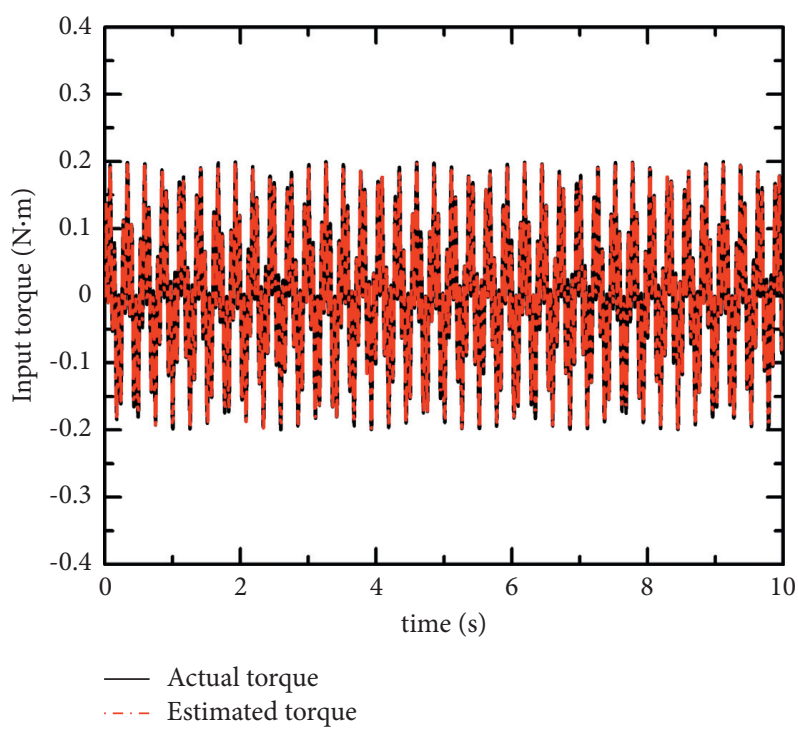

FIGURE 14: Time histories of the input torque (multifrequency excitation).

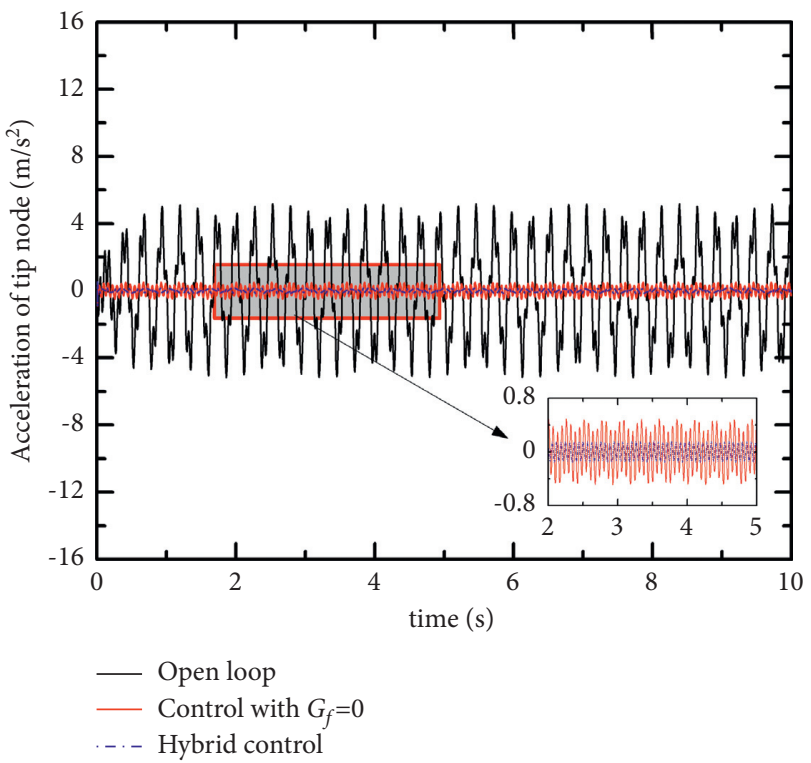

Figure 15: Time histories of acceleration at the tip node (multifrequency excitation). 


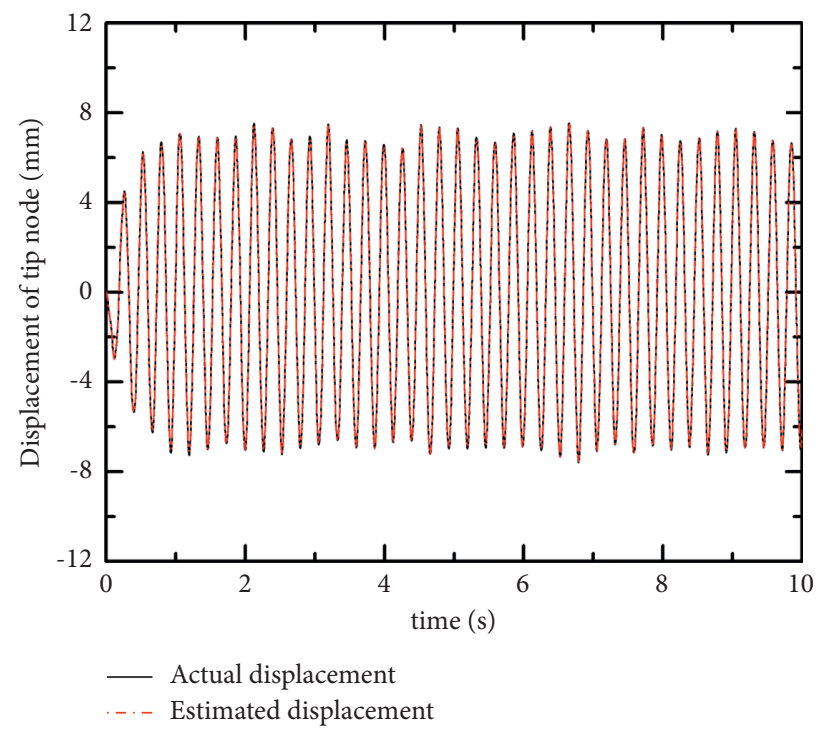

FIgURE 16: Time histories of displacements at the tip node (excitation with white noise).

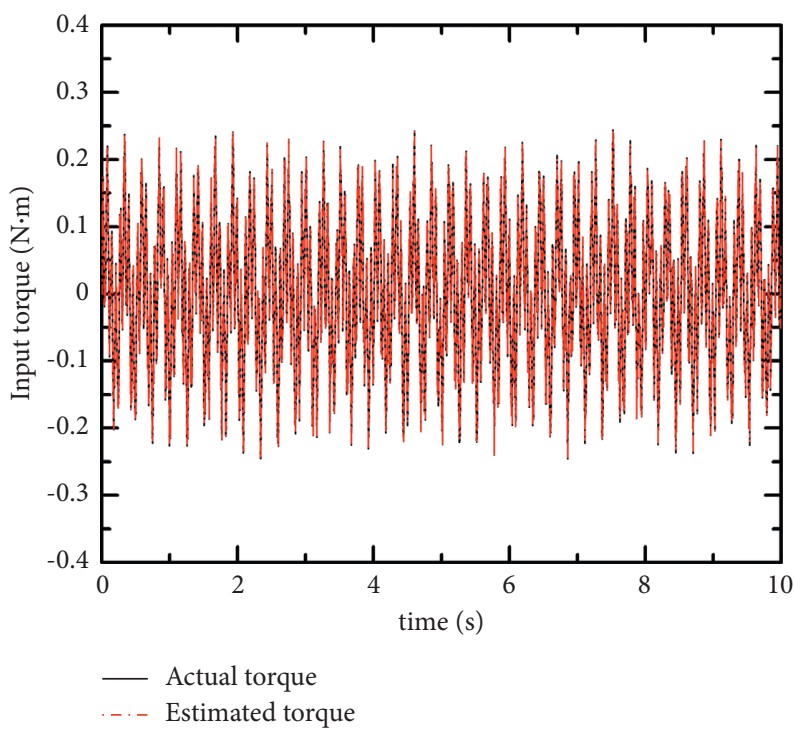

Figure 17: Time histories of the input torque (excitation with white noise).

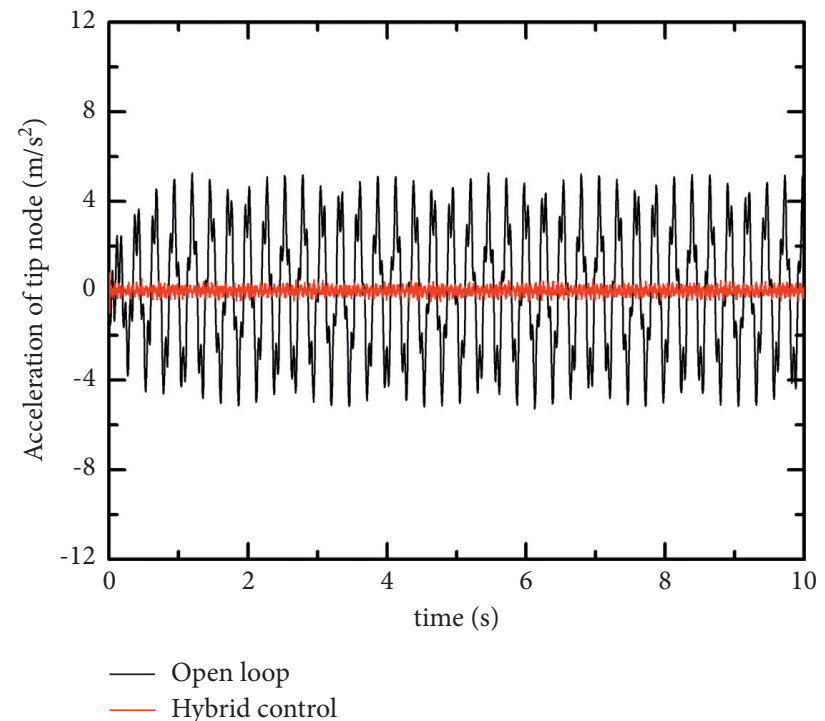

FIGURE 18: Time histories of acceleration at the tip node (excitation with white noise). 


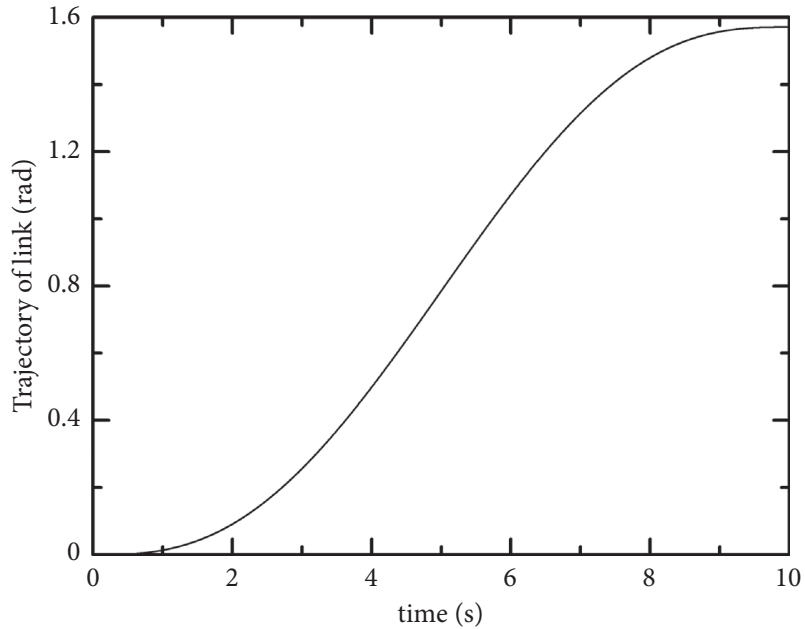

FIgURE 19: Time histories of the quantic polynomial trajectory.

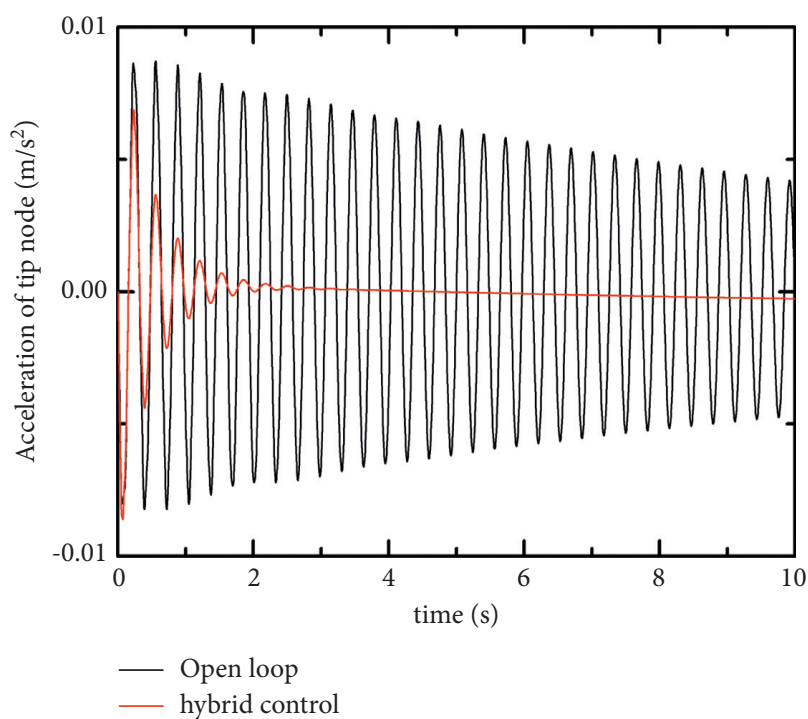

Figure 20: Time histories of the acceleration at the tip node (quantic polynomial trajectory).

\section{Conclusion}

In this paper, a time-delayed acceleration feedback controller for reducing the vibration of a single-link flexible manipulator is presented. An augmented discretized delayfree state derivate space system is established by employing a discrete transformation and random-walk process. A hybrid controller that uses a combination of the feedback signal and feedforward one is designed to cancel out external excitation and damp out vibration at the same time. Compared with the conventional LQG method, a discrete acceleration-based Kalman filtering method is developed to estimate the system states and external excitation with good accuracy for the improved control behavior, regardless of the effect of white noise. Moreover, it can be further confirmed from simulations that the introduction of time delay will become a favorable factor in vibration suppression, and the control performance will be improved as the time delay is chosen within a reasonable range. The simulation results demonstrated the effectiveness of the proposed control scheme. This study provides a basis for validating its practical feasibility, which is an open research topic.

\section{Data Availability}

The data used to support the findings of this study are available from the corresponding author upon request.

\section{Conflicts of Interest}

The authors declare that they have no conflicts of interest.

\section{References}

[1] Z. Liu, X. Tang, and L. Wang, "Research on the dynamic coupling of the rigid-flexible manipulator," Robotics and Computer-Integrated Manufacturing, vol. 32, pp. 72-82, 2015.

[2] H. N. Rahimi and M. Nazemizadeh, "Dynamic analysis and intelligent control techniques for flexible manipulators: a review," Advanced Robotics, vol. 28, no. 2, pp. 63-76, 2014.

[3] C. T. Kiang, A. Spowage, and C. K. Yoong, "Review of control and sensor system of flexible manipulator," Journal of Intelligent and Robotic Systems, vol. 77, no. 1, pp. 187-213, 2015.

[4] K. Lochan, B. K. Roy, and B. Subudhi, "A review on two-link flexible manipulators," Annual Reviews in Control, vol. 42, pp. 346-367, 2016.

[5] Z. Mohamed, M. Sayahkarajy, and A. A. Mohd Faudzi, "Review of modelling and control of flexible-link manipulators," Proceedings of the Institution of Mechanical Engineers-Part I: Journal of Systems \& Control Engineering, vol. 230, no. 8, pp. 861-873, 2016.

[6] M. O. Tokhi, Z. Mohamed, and M. H. Shaheed, "Dynamic modelling of a flexible manipulator system incorporating payload: theory and experiments," Journal of Low Frequency Noise, Vibration and Active Control, vol. 19, no. 4, pp. 209229, 2000.

[7] S. Yu and M. A. Elbestawi, "Modelling and dynamic analysis of a two-link manipulator with both joint and link flexibilities," Journal of Sound and Vibration, vol. 179, no. 5, pp. 839-854, 1995.

[8] H. Karagülle, L. Malgaca, M. Dirilmiş, M. Akdağ, and S. Yavuz, "Vibration control of a two-link flexible manipulator," Journal of Vibration and Control, vol. 23, no. 12, pp. 2023-2034, 2015.

[9] Q.-X. Meng, X.-Z. Lai, Y.-W. Wang, and M. Wu, "A fast stable control strategy based on system energy for a planar singlelink flexible manipulator," Nonlinear Dynamics, vol. 94, no. 1, pp. 615-626, 2018.

[10] M. Sayahkarajy, Z. Mohamed, A. A. M. Faudzi, and E. Supriyanto, "Hybrid vibration and rest-to-rest control of a two-link flexible robotic arm using Ho loop-shaping control design," Engineering Computations, vol. 33, no. 2, pp. 395-409, 2016.

[11] X. Yang, S. S. Ge, and W. He, "Dynamic modelling and adaptive robust tracking control of a space robot with twolink flexible manipulators under unknown disturbances," International Journal of Control, vol. 91, no. 4, pp. 969-988, 2018.

[12] U. Steve, J. Z. Sasiadek, and I. Barkana, "Modeling and direct adaptive control of a flexible-joint manipulator," Journal of 
Guidance, Control, and Dynamics, vol. 35, no. 1, pp. 25-39, 2012.

[13] B. Luo, H. Huang, J. Shan, and H. Nishimura, "Active vibration control of flexible manipulator using auto disturbance rejection and input shaping," Proceedings of the Institution of Mechanical Engineers - Part G: Journal of Aerospace Engineering, vol. 228, no. 10, pp. 1909-1922, 2014.

[14] C. d. Castri and A. Messina, "Exact modeling for control of flexible manipulators," Journal of Vibration and Control, vol. 18, no. 10, pp. 1526-1551, 2012.

[15] R. Kumar, "Effective active vibration control of single link flexible manipulator with modified positive position feedback control in the presence of instrumentation phase lead/lag," Journal of Vibration and Control, vol. 19, no. 10, pp. 15381560, 2013.

[16] Z. C. Qiu, "Acceleration sensor based vibration control for flexible robot by using PPF algorithm," in Proceedings of the IEEE International Conference on Control and Automation, ICCA, pp. 1335-1339, Guangzhou, China, June 2007.

[17] P. T. Kotnik, S. Yurkovich, and Ü. Özgüner, "Acceleration feedback for control of a flexible manipulator arm," Journal of Robotic Systems, vol. 5, no. 3, pp. 181-196, 2010.

[18] G. R. Chen, A. Desages, and P. Julian, "Trajectory tracking and robust stability for a class of time-delayed flexible-joint robotic manipulators," International Journal of Control, vol. 68, no. 2, pp. 259-276, 1997.

[19] S. Y. Jiang, "Oscillation suppression in a delay-coupled flexible-joint system," Applied Mechanics and Materials, vol. 477-478, pp. 123-127, 2014.

[20] S.-Y. Jiang, J. Xu, and Y. Yan, "Stability and oscillations in a slow-fast flexible joint system with transformation delay," Acta Mechanica Sinica, vol. 30, no. 5, pp. 727-738, 2014.

[21] J. Xu and S. Jiang, "Delay-induced Bogdanovu-Takens bifurcation and dynamical classifications in a slow-fast flexible joint system," International Journal of Bifurcation and Chaos, vol. 25, no. 9, pp. 87-118, 2015.

[22] S. R. Li and H. Q. Yin, "Adaptive position/force control for coordinated multiple flexible-joint manipulators with time delay," Control Theory \& Applications, vol. 34, no. 9, pp. 1208-1214, 2017.

[23] Z. C. Qiu and C. J. Wu, "Time-delayed vibration control of a rotating flexible manipulator based on model output prediction feedback," Journal of Aerospace Engineering, vol. 29, no. 4, 2015.

[24] A. Jnifene and A. Fahim, "A computed torque/time delay approach to the end-point control of a one-link flexible manipulator," Dynamics and Control, vol. 7, no. 2, pp. 171-189, 1997.

[25] N. A. Saeed and W. A. El-Ganaini, "Utilizing time-delays to quench the nonlinear vibrations of a two-degree-of-freedom system," Meccanica, vol. 52, no. 11, pp. 2969-2990, 2017.

[26] N. A. Saeed, G. M. Moatimid, F. M. F. Elsabaa, and Y. Y. Ellabban, "Time-delayed control to suppress a nonlinear system vibration utilizing the multiple scales homotopy approach," Archive of Applied Mechanics, vol. 91, no. 3, pp. 1193-1215, 2021.

[27] F. An, W.-d. Chen, and M.-q. Shao, "Dynamic behavior of time-delayed acceleration feedback controller for active vibration control of flexible structures," Journal of Sound and Vibration, vol. 333, no. 20, pp. 4789-4809, 2014.

[28] F. An, W.-D. Chen, and M.-Q. Shao, "Study on discrete acceleration feedback control with time delay," Journal of Vibration and Control, vol. 21, no. 7, pp. 1267-1285, 2015.
[29] F. Bellezza, L. Lanari, and G. Ulivi, "Exact modeling of the flexible slewing link," IEEE International Conference on Robotics and Automation, vol. 1, pp. 734-739, 1990.

[30] M. Shao, Y. Huang, and V. V. Silberschmidt, "Intelligent manipulator with flexible link and joint: modeling and vibration control," Shock and Vibration, vol. 2020, Article ID 4671358, 15 pages, 2020.

[31] H. Su, J. Chu, and S. Wang, "A control algorithm for a class of discrete time-delay systems based on staged transformation and its application," IFAC Proceedings Volumes, vol. 29, no. 1, pp. 1014-1019, 1996.

[32] S. H. Park, P. S. Kim, O.-K. Kwon, and W. H. Kwon, "Estimation and detection of unknown inputs using optimal FIR filter," Automatica, vol. 36, no. 10, pp. 1481-1488, 2000.

[33] C. K. Chui, Kalman filtering: with real-time applications, Springer, New York, NY, USA, 2010.

[34] C. Ho and C. Ma, "Active vibration control of structural systems by a combination of the linear quadratic Gaussian and input estimation approaches," Journal of Sound and Vibration, vol. 301, no. 3, pp. 429-449, 2007.

[35] Y.-l. Yang, Y.-d. Wei, J.-q. Lou, L. Fu, and X.-w. Zhao, "Nonlinear dynamic analysis and optimal trajectory planning of a high-speed macro-micro manipulator," Journal of Sound and Vibration, vol. 405, no. 1, pp. 112-132, 2017. 